\title{
Tear Film Osmolarity in Response to Long-Term Orthokeratology Treatment
}

\author{
Amelia Nieto-Bona, Ph.D., María Nombela-Palomo, M.Sc., \\ Gema Felipe-Márquez, M.Sc., and Miguel A. Teus, Ph.D.
}

\begin{abstract}
Purpose: To compare tear film osmolarity (TFO) measurements in noncontact lens (CL) wearers and wearers of hydrogel or overnight orthokeratology (OK) CLs, and to assess possible effects of long-term OK on TFO. Methods: Overall, 108 subjects with moderate myopia participated in 2 experiments, and TFO was measured using the TearLab osmolarity system. In experiment 1 , TFO measurements were made in 77 right eyes of 23 nonCL wearers, 26 hydrogel wearers, and 28 OK wearers. Subjects in the last 2 groups had worn their CL for at least 3 years. In experiment 2, 31 individuals (habitual soft CL wearers) were enrolled for prospective longterm follow-up of OK treatment. These subjects were fitted with ParagonCRT $(n=16)$ or Seefree $(n=15)$ lenses, and TFO readings were taken at baseline and after 1 month and 1 year of lens wear and after 1 month of OK treatment interruption.
\end{abstract}

Results: Values of TFO were within the normal limits in all 3 subject groups, although significantly lower osmolarities $(P<0.01)$ were observed in non-CL wearers $(281.7 \pm 5.9 \mathrm{mOsm} / \mathrm{L})$ compared with hydrogel $(291 \pm 16.5 \mathrm{mOsm} / \mathrm{L})$ or OK lens wearers $(301.7 \pm 10.8 \mathrm{mOsm} / \mathrm{L})$. In experiment 2, TFO differed significantly at baseline between the Paragon-CRT and Seefree groups $(P<0.05)$, and a significant decrease in TFO compared with baseline $(P<0.01)$ was observed in the Paragon-CRT group after 1 month of cessation of lens wear.

Conclusion: Higher TFO values were observed in lens wearers (hydrogel or $\mathrm{OK}$ ) than non-CL wearers. After interruption of OK treatment, TFO returned to similar values to those found in non-CL wearers.

Key Words: Tear film osmolarity—Overnight orthokeratology-Contact lenses-TearLab osmolarity system.

(Eye \& Contact Lens 2016;0: 1-6)

$\mathrm{O}$ rthokeratology (OK), also known as overnight corneal refractive therapy or corneal reshaping, is a clinical technique designed to transiently reduce or eliminate myopia. Over the years, developments in the field have made $\mathrm{OK}$ a feasible correction option for subjects who prefer not to wear spectacles or contact

From the Department of Optics II (Optometry and Vision), Faculty of Optics and Optometry (A.N.-B.), Contactology and Optometry Research Group (GICO), Complutense University of Madrid, Madrid, Spain; Department of Optics II (Optometry and Vision), Faculty of Optics and Optometry (M.N.-P., G.F.-M.), Complutense University of Madrid, Madrid, Spain; and Deparment of Surgery, University of Alcalá (M.A.T.), Madrid, Spain.

The authors have no funding or conflicts of interest to disclose.

Address correspondence to Amelia Nieto-Bona, Ph.D., Department of Optics II (Optometry and Vision), Faculty of Optics and Optometry, Complutense University of Madrid, Arcos de Jalon 118, Madrid 28037, Spain; e-mail: amnop@ucm.es

Accepted September 25, 2016.

DOI: 10.1097/ICL.0000000000000347 lenses (CLs) during the day. Today's fitting techniques enable predictable and safe outcomes for improving uncorrected visual acuity and contrast sensitivity, ${ }^{1-4}$ including satisfactory subjective vision according to the National Eye Institute Refractive Error Quality of Life Instrument-42 questionnaire. ${ }^{5}$

The success of OK has been accompanied by a need to address the corneal response to treatment. Recent research efforts have examined changes in corneal morphology and physiology by assessing the effects of treatment on cell density, ${ }^{6-8}$ thicknesses of the corneal layers, ${ }^{9-11}$ subbasal nerve plexus (SBNP) distribution, ${ }^{7,12}$ and corneal biomechanics. ${ }^{13-15}$ Some studies have also determined OK effects on tear film components, ${ }^{16}$ such as inflammatory mediators. ${ }^{17}$ These studies conducted both in animals ${ }^{18-20}$ and humans $s^{6,7,12}$ have identified corneal changes produced in stromal and epithelial cell density, SBNP thickness and distribution, ${ }^{7,12}$ and epithelial layer thickness ans also changes in the levels and distribution of proinflammatory mediators in the tear film samples of OK wearers. ${ }^{17,21}$ To date, however, the effects of OK treatment on tear film osmolarity (TFO) have not yet been examined. Some authors have reported that TFO increases are associated with CL wear. ${ }^{22,23}$ As suggested by Efron et al, ${ }^{24} \mathrm{CL}$ wear may increase osmolarity in two ways: (1) by inducing tear film evaporation and (2) by inducing electrolyte concentration changes. The cycle of events leading to elevated TFO has been described as a closed loop in a report by the Dry Eye Workshop (DEWS). ${ }^{25}$ According to this theory, hyperosmolarity causes damage to the corneal epithelial surface by activating a cascade of inflammatory events on the ocular surface, including the release of inflammatory mediators into the tears, which could be a cause of nerve injury and/or raised TFO.

This study was designed to examine the TFO response to OK wear. In a first experiment, we compared TFO in OK and hydrogel CL wearers versus non-CL wearers, and then in experiment 2 , we examined TFO changes produced in response to long-term $\mathrm{OK}$ treatment. We also determined whether these changes were reversible after treatment cessation.

\section{MATERIAL AND METHODS}

The study was conducted by the Contactology and Optometry Research Group, Complutense University of Madrid (Spain). The study protocol adhered to the tenets of the Declaration of Helsinki and was approved by the Ethics Committee of the Hospital San Carlos (Madrid, Spain). Participants gave their written informed consent.

\section{Study Design}

The study was designed as two experiments. The first (experiment 1) was a case/control study comparing TFO data in non-CL 
wearers and long-term wearers ( $\geq 3$ years) of two CL types. For these measurements, 77 right eyes of 77 subjects were divided into 3 groups: (1) non-CL wearers, (2) hydrogel lens wearers, and (3) OK lens wearers. Experiment 2 was a prospective, longitudinal, single-center study designed to assess the TFO response to $\mathrm{OK}$ wear over time (13 months). In this experiment, TFO measurements were made in 31 right eyes of 31 individuals (habitual soft $\mathrm{CL}$ wearers) undergoing overnight $\mathrm{OK}$ for the first time. Measurements of TFO were then compared for baseline, 1 month and 1 year after starting OK treatment, and 1 month after OK treatment cessation. The subjects were assigned to two groups matched for age, refractive state, and corneal curvature and fitted with two OK lens designs (Seefree or Paragon-CRT).

Tear film osmolarity was measured using the TearLab system (TearLab Corp, San Diego, CA). We calculated sample sizes from published normality data (means, standard deviations, and coefficients of repeatability). ${ }^{25}$ For a significance (alpha) of 0.05 for a type I error and beta of 0.2 for a type II error in a 2-tailed test, the number of subjects needed for each group in experiment 1 was calculated as 21 to detect a difference greater than the standard deviation (assumed as 9 units or $\mathrm{mOsm} / \mathrm{L}$ ). For the second experiment, it was calculated that 15 subjects per treatment group would be needed to detect a significant difference greater than 9 units assuming the same criteria as for the above calculations and a correlation coefficient between the initial and final measurement of 0.75 .

\section{Participants}

All subjects enrolled were subjected to a full eye examination. Inclusion criteria for both groups of participants were as follows: age, 18 to 30 years; -0.50 to -5.00 diopters (D) of sphere, -0.25 to $-1.25 \mathrm{D}$ of cylinder, and monocular best-corrected visual acuity (BCVA) of at least 0.04 logarithm of the minimum angle of resolution ( $\log M A R)$. The baseline refractive state of each participant was measured as manifest refraction using a phoropter at the study outset.

Subjects were excluded if they had used gas permeable CL in the past. In experiment 1 , hydrogel and OK lens wearers continued using their $\mathrm{CL}$ as normal. In experiment 2, participants were instructed to stop wearing their soft CLs 4 weeks before the study start.

Subjects were also excluded if they were pregnant or planning to become pregnant over the following 12 months. Individuals who experienced any systemic or eye disease including dry eye syndrome (DES) were also excluded because this could affect TFO. Following the DEWS ${ }^{25}$ recommendations, both symptom questionnaires and objective clinical measures were used as diagnostic criteria of DES. These tests were fluorescein breakup time (FBUT) for tear film stability, ${ }^{26}$ the phenol red test (PRT) for tear secretion, ${ }^{27}$ and McMonnies questionnaire ${ }^{28}$ for ocular symptoms and their severity. The cutoffs used for DES were FBUT of $<10$ seconds, PRT of $<18 \mathrm{~mm}$, and McMonnies of $>10$ points.

\section{Experiment 1}

Two visits were necessary to complete all measurements. Out of 90 individuals enrolled, 13 were excluded: 9 did not attend the second visit, and in 4 subjects, TFO data could not be obtained because of the limitations of the instrument. This left a final study population of 77 subjects: 26 non-CL wearers, 23 hydrogel lens wearers, and 28 OK (Paragon-CRT) lens wearers. Participants in the hydrogel group had been wearing hydrogel daily disposable lenses for at least 3 years before the study outset. It was also ensured that they wore their lenses at least 6 days per week and that the hydrogel lenses were composed of the same material $(60 \%$ omafilcon A). These participants were recruited among the patients visiting the Optometry Clinic of the Complutense University. NonCL wearers were recruited among the undergraduates of the optometry degree course of this university. The OK lens wearers recruited were participants in a prior study conducted by our group and had been wearing Paragon-CRT lenses also for at least 3 years. ${ }^{7,29}$ All subjects were whites.

\section{Experiment 2}

Of 40 white subjects enrolled through the Web page (http:// www.ucm.es/accion-social), 4 refused to participate and 5 withdrew after starting OK treatment, leaving a final population of 31 subjects. These subjects were divided into 2 groups matched for age, refractive error, and corneal curvature $(P>0.05)$ to be fitted with Paragon-CRT $(n=16)$ or Seefree $(n=15)$ OK lenses. Apart from the visits for the initial assessment and TFO measurements, these participants were required to attend standard visits for $\mathrm{OK}$ lens fitting. The 2 lens designs were HDS 100 Paragon-CRT (Paragon Vision Sciences; Interlenco, Madrid, Spain) and Seefree made of Boston $\mathrm{XO}_{2}$ material (Conóptica, Barcelona, Spain). Both designs are double, reverse geometry generation, advanced $\mathrm{OK}$ lenses.

\section{Procedures}

\section{Initial Examination}

The study followed a controlled protocol. All measurements were made in the same office. In the first visit, clinical tests were conducted in the same order by the same clinician for all subjects enrolled: corneal topography, visual acuity (using Early Treatment Diabetic Retinopathy Study logMAR charts), manifest refraction, PRT, FBUT, and McMonnies questionnaire. Keratometric measurements were performed with the Atlas 9000 topographer (Carl Zeiss, Jena, Germany). Slitlamp examination included the assessment of the ocular surface system (cornea, conjunctiva, eyelid, and lid margin) to rule out anterior or posterior blepharitis, conjunctivitis, and meibomian gland dysfunction or any abnormality that could influence the results or contraindicate OK treatment. Meibomian gland expressibility was checked by applying pressure with the finger on the central tarsus of the lower lid. Corneal integrity was confirmed in a fluorescein examination.

\section{Tear Film Osmolarity Measurements}

The TearLab Osmolarity System (TearLab) is easy to use as suggested by Tomlinson et $\mathrm{al}^{30}$ and shows good agreement with other instruments. According to the DEWS report, ${ }^{24}$ osmolarity is considered among the most objective assessments of dry eye disease. ${ }^{31} \mathrm{We}$ followed the measurement procedure described by others. $^{31}$ To ensure the system was working normally, we used single-dose saline in every study visit before the measurements. Three consecutive TFO readings were obtained for this purpose, as suggested by Khanal and Millar and Tomlinson et al. ${ }^{32,33}$ After taking TFO readings, the tear film was examined by slitlamp microscopy to rule out any adverse events that could affect outcomes. In the OK groups, measurements were made in the morning 
TABLE 1. Descriptive Variables Recorded in the 108 Study Participants at Baseline ${ }^{a}$

\begin{tabular}{|c|c|c|c|c|c|c|c|}
\hline \multirow[b]{3}{*}{ Group } & \multicolumn{4}{|c|}{ Experiment 1} & \multicolumn{3}{|c|}{ Experiment 2 (Data Obtained at Baseline) } \\
\hline & Paragon-CRT, $(n=28)$ & $\mathrm{H} \mathrm{CL},(n=23)$ & No $C L,(n=26)$ & \multirow[b]{2}{*}{ ANOVA, $P$} & Paragon-CRT, $(n=16)$ & Seefree, $(n=15)$ & \multirow[b]{2}{*}{ ANOVA, $P$} \\
\hline & & Mean \pm SD & & & \multicolumn{2}{|c|}{ Mean \pm SD } & \\
\hline Male/female, $\mathrm{n}$ & $16 / 12$ & $10 / 13$ & $14 / 12$ & & $7 / 9$ & $6 / 9$ & \\
\hline Age, yrs & $23.6 \pm 3.1$ & $22.8 \pm 1.8$ & $23.6 \pm 1.6$ & 0.39 & $23.9 \pm 1.6$ & $24.2 \pm 1.2$ & 0.61 \\
\hline $\mathrm{SE}$, diopters & $-1.90 \pm 1.00$ & $-2.31 \pm 0.80$ & $-1.90 \pm 0.92$ & 0.22 & $-2.10 \pm 0.50$ & $-1.76 \pm 0.96$ & 0.12 \\
\hline Sim Kflat, diopters & $43.51 \pm 1.26$ & $43.19 \pm 1.50$ & $43.26 \pm 1.35$ & 0.69 & $43.21 \pm 1.40$ & $43.51 \pm 1.26$ & 0.53 \\
\hline Sim Ksteep, diopters & $44.14 \pm 1.42$ & $43.92 \pm 1.56$ & $44.02 \pm 1.48$ & 0.87 & $43.94 \pm 1.46$ & $44.14 \pm 1.42$ & 0.70 \\
\hline$B C V A, \log M A R$ & $-0.06 \pm 0.06$ & $-0.12 \pm 0.05$ & $-0.10 \pm 0.06$ & $<0.01$ & $-0.10 \pm 0.06$ & $-0.12 \pm 0.05$ & 0.22 \\
\hline FBUT, sec & $9.6 \pm 2.10$ & $9.53 \pm 3.00$ & $13.9 \pm 7.00$ & $<0.01$ & $9.9 \pm 2.00$ & $9.7 \pm 3.00$ & 0.82 \\
\hline Phenol red test, $\mathrm{mm}$ & $17.2 \pm 5.10$ & $19.8 \pm 6.20$ & $23.7 \pm 1.60$ & $<0.01$ & $18.4 \pm 5.60$ & $19.1 \pm 5.90$ & 0.74 \\
\hline McMonnies Q & $7.9 \pm 5$ & $5.8 \pm 4$ & $4.1 \pm 3$ & $<0.01$ & $7 \pm 3$ & $7.47 \pm 3$ & 0.67 \\
\hline
\end{tabular}

${ }^{a} \mathrm{CL}$, contact lenses; FBUT, fluorescein breakup time; H, hydrogel; SD, standard deviation; SE, sphere equivalent; Sim Kflat and Sim Ksteep, simulated corneal curvature readings for the flattest and steepest meridians; BCVA, best-corrected visual acuity; logMAR, logarithm of the minimum angle of resolution; ANOVA, analysis of variance.

2 to 4 hours after removing the lenses. In the soft CL wearers in experiment 1 , measurements were made within 2 to 4 hours of waking without participants using their lenses. All subjects were also asked to not use eyedrops.

\section{Orthokeratology Lens Fitting}

The following adaptation procedure for the lenses was followed by a single CL practitioner according to the lens manufacturers' protocols: (1) lens specifications were determined using the calculation rule (Paragon-CRT) or from the calculation mode of the Oculus Easygraph (Oculus Optikgeraete GMBH) (Seefree; Conóptica); (2) adequate fitting was assessed using fluorescein; and (3) satisfactory fitting was confirmed by the typical bull's-eye pattern observed by corneal topography after an overnight trial.

For lens fitting, subjects were examined 1 day, 1 week, and 1 month after starting OK treatment. Subjects were instructed to sleep about 8 hours with their OK lenses every night and to clean them on a daily basis. All participants used MeniCare Plus solution and a weekly protein remover (Menicon Progent; Menicon, Co, Ltd, Nagoys, Japan). They were also supplied with single doses of preservative-free lubricant eyedrops (Moisture drops; Avizor, Madrid, Spain) for use before inserting the lenses in their eyes every night and before lens removal every morning. The use of the same lubricant was designed to avoid possible composition effects on TFO. Benelli et $\mathrm{al}^{31}$ found that some lubricant eyedrops reduced osmolarity more than others after instillation.

\section{Data Analysis}

Statistical tests were performed using the STATGRAPHICS package Centurion XVI, version 16.1.17. Only data obtained in right eyes were used in this study. In both experiments, the TFO data showed a Gaussian distribution according to the KolmogorovSmirnov test. In Experiment 1, data between groups were compared by one-way analysis of variance (ANOVA). In Experiment 2, we compared TFO over time through repeated measurements analysis and compared results between the groups (Paragon-CRT, Seefree) using an unpaired-sample $t$ test. To analyze TFO over time when both OK groups were combined, we used 1-factor repeated measures analysis of variance. Significance was set at $P<0.05$.

\section{RESULTS}

In Table 1, we provide baseline means and standard deviations for age, BCVA, refractive state, and corneal curvature measurements for the right eyes of the participants of both experiments ( $n=77$ and $n=31$, respectively). None of the OK lens wearers who completed the treatment experienced adverse effects related to lens wear, and no abnormalities were detected by slitlamp microscopy during the follow-up. Table 1 also provides baseline means and standard deviations for the BUT, PRT, and McMonnies questionnaire results. None of the participants had DES according to diagnostic criteria established in Material and Method section.

\section{Experiment 1}

Values of TFO were within normal limits in all three groups (Fig. 1). One-way analysis of variance identified significant differences in TFO among groups $(P<0.01 ; \mathrm{F}=28.10)$ : higher TFO values were observed in $\mathrm{OK}$ and hydrogel lens wearers than in non-CL wearers $(P<0.01)$ and values were higher in the OK group than in hydrogel group $(P<0.05)$.

\section{Experiment 2}

Mean sphere magnitudes and sphere equivalents decreased significantly during $\mathrm{OK}$ wear $(P<0.00)$, whereas refractive

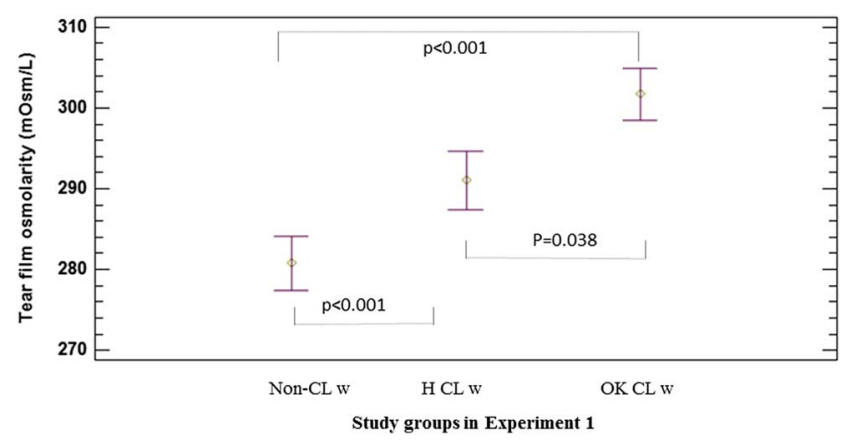

FIG. 1. Tear film osmolarity (in $\mathrm{mOsm} / \mathrm{L}$ ) values recorded in noncontact lens wearers, hydrogel lens wearers, and orthokeratology lens wearers (experiment 1 ). $\mathrm{H}=$ hydrogel. Error bars indicate standard deviation at the $95 \%$ confidence level. Non-contact lens wearers showed significantly lower tear film osmolarity values than other two groups (analysis of variance, $P=0.0000$; Levene post hoc $P$ values indicated in the figure). 
cylinder values remained unchanged in both the Paragon-CRT and Seefree groups. Uncorrected visual acuity significantly improved in both OK groups $(P<0.00)$. After 1 year of $\mathrm{OK}$ treatment, central corneal power was significantly reduced at both the steeper and flatter meridians $(P<0.00)$ in the two groups. After discontinuation of treatment, all measurements returned to baseline.

Measurements of TFO were within normal limits during followup in both OK treatment groups. Means and standard deviations for the TFO values recorded at each time point in both groups are provided in Figure 2. Osmolarities differed significantly at baseline between the Paragon-CRT and Seefree groups (299.9 \pm 12.7 vs. $288.5 \pm 9.5 \mathrm{mOsm} / \mathrm{L} ; \quad P=0.02 ; \mathrm{F}=6.01 ; \quad$ Fig. 2A). Through repeated-measures analysis, significant differences in TFO were detected over time in the Paragon-CRT group, and TFO was significantly lower at the final time point after lens wear cessation than at the remaining time points $(P<0.01 ; \mathrm{F}=4.75)$. Readings of TFO were also reduced in the Seefree lens wearers in the final follow-up session, but the difference was not significant $(P>0.05 ; \mathrm{F}=1.81$, Fig. 2A). When the TFO data for both groups were combined (Fig. $2 \mathrm{~B}$ ), analysis of variance also revealed a significant change in TFO over time $(P<0.01 ; \mathrm{F}=5.32)$, and the post hoc rank test identified a significant difference between the TFO value recorded 1 month after OK lens discontinuation compared with the remaining time points (baseline, I month, and 1 year) (Fig. 2B).

\section{DISCUSSION}

Orthokeratology is an effective reversible treatment that frees the patient from the need for spectacles or CLs during waking hours. Despite numerous investigations assessing the response of eyes to this treatment, to the best of our knowledge, no study has addressed the impact of OK lens wear on TFO. This study compares TFO measurements in non-CL wearers and long-term (3 years or longer) hydrogel and OK lens wearers. In addition, TFO changes produced during long-term (1 year) OK treatment and the reversibility of such changes after treatment cessation were assessed for the first time in young adults wearing two different OK lens designs.

In a meta-analysis, Tomlinson et $\mathrm{al}^{33}$ established normal TFO at $302 \mathrm{mOsmol} / \mathrm{L}(275-316 \mathrm{mOsmol} / \mathrm{L})$ and the cutoff for tear film hyperosmolarity of the human cornea at $316 \mathrm{mOsmol} / \mathrm{L}$. In our study, all TFO measurements were within the normal limits. Lowest and highest TFO values were found respectively in non-CL wearers and $\mathrm{OK}$ lens wearers.

Although none of the TFO values recorded in our study could be considered hyperosmotic, we observed significantly higher osmolarities in CL wearers (hydrogel or OK) than non-CL wearers. Thus, it is reasonable to assume that this higher TFO could be attributed to CL wear.

These findings are in agreement with those of Sarac et $\mathrm{al}^{34}$ who noted increased TFO in wearers of two different types of daily disposable lenses (hydrogel and silicone hydrogel) over short periods. Osmolarities were similar to our values when measured before CL insertion (293 \pm 10.97 and $294 \pm 13.66 \mathrm{mOsmol} / \mathrm{L})$. Alghamdi et $\mathrm{al}^{35}$ recently investigated the effects of the duration of soft $\mathrm{CL}$ wear on the ocular surface system. Our measurements of TFO in hydrogel CL wearers are consistent with those obtained in their study for a similar period of CL wear. These authors observed no effects on TFO based on the type of hydrogel lens worn, that is, silicone hydrogel versus conventional hydrogel. However, their TFO values in non-CL wearers were higher, and they observed no significant differences between non-CL wearers and soft CL wearers as noted here. Given that the mean age of their non-CL wearer group was similar to ours, this difference between the studies could be attributable to methodological discrepancies, such as the time measurements were made (not specified in their study) or the fact that we took three consecutive TFO readings. In our study, wearing both CL types for at least 3 years raised TFO, although the final reading was lower than the cutoff for dry eye disease. Another finding was that the OK lens group showed significantly higher TFO values than the hydrogel lens group. We attribute this difference to greater mechanical forces exerted by the overnight lenses on the anterior corneal surface especially considering that measurements were taken in the morning when the tear film is more unstable because of these forces. Choy et $\mathrm{al}^{16}$ reported effects on tear composition, such as marked elevations in albumin and lactate dehydrogenase, indicating hypoxic stress to the eye surface during overnight $\mathrm{OK}$ lens wear. In addition, Gonzalez-Perez et $\mathrm{al}^{17}$ observed increased levels of inflammatory markers in subjects wearing OK lenses compared with the continuous wear of silicone hydrogel lens in the long term. This response could be explained
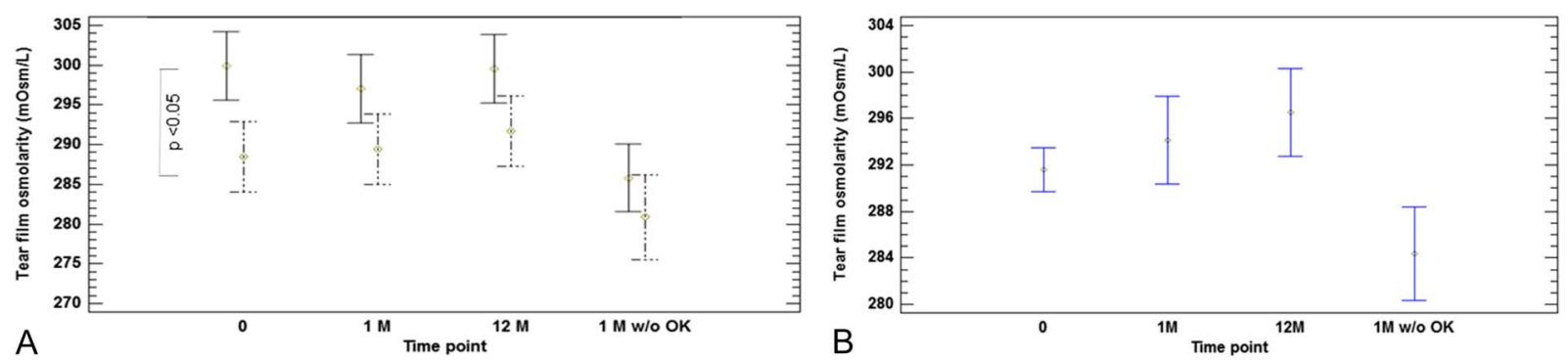

FIG. 2. Changes in tear film osmolarity ( $\mathrm{mOsm} / \mathrm{L}$ ) recorded during the 13-month orthokeratology (OK) trial (experiment 2). (A) Values of tear film osmolarity (TFO) plotted over time for the Paragon-CRT versus Seefree group. Unbroken line indicates Paragon-CRT group (analysis of variance; $P=0.005$ ). Values of TFO were significantly lower at 1 month without OK versus all the other time points. Broken line indicates Seefree group (analysis of variance, $P=0.1644$ ). Analysis of variance, Paragon-CRT versus Seefree at baseline $(P=0.022)$. (B) Mean TFO values plotted over time for the Paragon-CRT and Seefree groups combined. Error bars indicate standard deviation at the $95 \%$ confidence level. $M$, month, w/o OK, after interruption of OK treatment. $P=0.019$, analysis of variance. Values of TFO were significantly lower at 1 month without OK versus all the other time points $(P<0.05$ for each of the 3 comparisons). 
by the physical impact of treatment on the corneal epithelium. According to Liu et al, ${ }^{36}$ the corneal epithelium reacts to hyperosmolar stress, and this may occur in areas where the tear film is more unstable, like local thinner areas, as in OK wearers. However, the slightly higher TFO detected here in the OK lens wearers compared with the subjects who habitually wore hydrogel is not likely to produce any negative effects. The DEWS ${ }^{25,37}$ reported that an elevated TFO is one of the key pathological factors in dry eye, leading to ocular discomfort associated with damage to the ocular surface and inflammation. In contrast, Carracedo et $\mathrm{al}^{21}$ noted that OK treatment did not produce symptoms of ocular dryness, possibly because their OK subjects who did not wear CL during the day were compared with wearers of rigid gaspermeable CL who reported symptoms of discomfort. The lack of a daytime wearing regimen could thus be a benefit for OK lens wearers in that discomfort arising from hyperosmolarity is avoided. In effect, Liu et $\mathrm{al}^{36}$ suggested that blinking and reflex secretion of tears should serve to mitigate prolonged local hyperosmotic conditions, as probably happens in OK wearers throughout the day. It would be interesting to confirm this notion in OK patients. Other studies have shown an interrelationship between SBNP density and corneal sensitivity. Nieto-Bona et $\mathrm{al}^{7}$ reported reduced SBNP nerve densities in response to $\mathrm{OK}$ treatment. Lum et $\mathrm{al}^{12}$ also described SBNP nerve fiber redistribution in OK lens wearers, whereas Lin$\mathrm{na}^{38}$ directly correlated subbasal nerve morphology changes with corneal sensitivity in patients who had undergone laser in situ keratomileusis surgery. Nerve injury has been shown to reduce corneal sensitivity, which in turn increases TFO. ${ }^{25}$ This could explain the higher TFO values recorded in our OK group. Correlating these three variables would be another interesting topic for future studies.

In the second experiment of our study, we compared TFO values between two groups of subjects (all prior CL wearers) fitted with different OK lens designs over time. Unexpectedly, these two groups differed significantly in terms of TFO at baseline, with the Paragon-CRT group showing the higher mean value. When trying to find an explanation for this difference, we noted similar distributions of means and standard deviations and similar trends in this variable over time in the two groups. Therefore, we propose two possible explanations: (1) the wash-out period was not long enough or (2) the subjects failed to comply with the stipulated wash-out period. We believe that this second factor was the main reason for the higher osmolarity observed in the Paragon-CRT group at baseline. In addition, when changes in TFO were examined over time, the greater decrease observed at the final time point in the Paragon-CRT group was likely attributable to the greater starting value $(P<0.01 ; \mathrm{F}=4.75)$. We propose that the subjects in this group did not adhere as much to the 4-week wash-out period, explaining their higher baseline TFO value. Because of this situation, when approaching the end of the treatment period, we purposely stressed the importance of complying with the 1-month period of non-OK lens wear for the final TFO readings. Another remarkable finding was that TFO did not significantly increase during OK lens wear, and only slight fluctuations were observed over the year of treatment. In a clinical assessment of the OculoSense Tear Lab instrument, Eperjesi et $\mathrm{al}^{39}$ argued that the difference in measurements that could be considered clinically relevant for healthy subjects is $33 \mathrm{mOsmol} / \mathrm{L}$. This difference is far from the trend observed here. Our data indicate a significant decrease in
TFO during OK lens wear and that recovered TFO values after 1 month of OK treatment cessation were similar to the TFO readings obtained in the non-CL wearers of experiment 1 . This suggests that the wash-out period was long enough and that the higher osmolarities observed at baseline in one of the $\mathrm{OK}$ treatment groups in experiment 2 could reflect TFO variability among subjects, a lack of compliance regarding the wash-out period before treatment, or the limitation of the measurement method. We should nevertheless underscore that all TFO values obtained here were within the normal limits for healthy corneas.

Thus, it seems that the presence of proinflammatory markers in the tear film, as reported by Gonzalez-Perez et $\mathrm{al}^{17}$ for a similar treatment period, does not raise TFO values beyond normal limits. It would be interesting to address the proinflammatory marker response in parallel with corneal epithelial and SBNP responses in the long term.

As far as we are aware, the present study is the first to determine TFO values during hydrogel and OK lens wear in young adults with special attention paid to the impacts on this variable of longterm OK treatment and the reversibility of these impacts after treatment cessation. In conclusion, our findings indicate that longterm hydrogel and OK lens wear leads to increased TFO values compared with non-CL (spectacle) wear, although the values observed here were still within the normal limits. In addition, TFO remained stable over 1 year of OK treatment, and by 1 month after treatment interruption, TFO had returned to lower than baseline levels. These low TFO levels were effectively comparable to the osmolarities observed in non-CL wearers and suggest the full reversibility of the corneal response to OK treatment.

\section{ACKNOWLEDGMENTS}

The authors thank Interlenco (Madrid, Spain) and Conóptica (Barcelona, Spain) for providing the orthokeratology lenses used in this study and Avizor (Madrid, Spain) for providing the lens cleaning solutions and lubricant eyedrops.

\section{REFERENCES}

1. Hiraoka T, Okamoto C, Ishii Y, et al. Time course of changes in ocular higher-order aberrations and contrast sensitivity after overnight orthokeratology. Invest Ophthalmol Vis Sci 2008;49:4314-4320.

2. Lorente-Velazquez A, Mesa AG, Gutierrez JR, et al. Long-term changes in straylight induced by corneal refractive therapy: a pilot study. Contact Lens Anterior Eye 2014;37:144-148.

3. Soni PS, Nguyen TT, Bonanno JA. Overnight orthokeratology: visual and corneal changes. Eye Contact Lens 2003;29:137-145.

4. Swarbrick HA. Orthokeratology review and update. Clin Exp Optom 2006; 89:124-143.

5. Queiros A, Villa-Collar C, Gutierrez AR, et al. Quality of life of myopic subjects with different methods of visual correction using the NEI RQL-42 questionnaire. Eye Contact Lens 2012;38:116-121.

6. Nieto-Bona A, Gonzalez-Mesa A, Nieto-Bona MP, et al. Short-term effects of overnight orthokeratology on corneal cell morphology and corneal thickness. Cornea 2011;30:646-654.

7. Nieto-Bona A, Gonzalez-Mesa A, Paz Nieto-Bona M, et al. Long-term changes in corneal morphology induced by overnight orthokeratology. Curr Eye Res 2011;36:895-904.

8. Zhong X, Chen X, Xie RZ, et al. Differences between overnight and longterm wear of orthokeratology contact lenses in corneal contour, thickness, and cell density. Cornea 2009;28:271-279.

9. Alharbi A, Swarbrick HA. The effects of overnight orthokeratology lens wear on corneal thickness. Invest Ophthalmol Vis Sci 2003;44:2518-2523.

10. Wang JFD, Simpson TL, Sorbara L, et al. Topographical thickness of the epithelium and total cornea after overnight wear of reverse-geometry rigid 
contact lenses for myopia reduction. Invest Ophthalmol Vis Sci 2003;44: 4742-4746.

11. Haque S, Fonn D, Simpson T, et al. Corneal and epithelial thickness changes after 4 weeks of overnight corneal refractive therapy lens wear, measured with optical coherence tomography. Eye contact lens 2004;30: 189-193. Discussion 205-186.

12. Lum E, Golebiowski B, Swarbrick HA. Mapping the corneal sub-basal nerve plexus in orthokeratology lens wear using in vivo laser scanning confocal microscopy. Invest Ophthalmol Vis Sci 2012;53:1803-1809.

13. Chen D, Lam AKC, Cho P. A pilot study on the corneal biomechanical changes in short-term orthokeratology. Ophthalmic Physiol Opt 2009;29: 464-471.

14. Gonzalez-Meijome JM, Villa-Collar C, Queiros A, et al. Pilot study on the influence of corneal biomechanical properties over the short term in response to corneal refractive therapy for myopia. Cornea 2008;27:421-426.

15. Nieto-Bona A, González-Mesa A, Villa-Collar C, et al. Biomechanical properties in corneal refractive therapy during adaptation period and after treatment interruption: a pilot study. J Optom 2012;5:164-170.

16. Choy CKM, Cho P, Benzie IFF, et al. Effect of one overnight wear of orthokeratology lenses on tear composition. Optom Vis Sci 2004;81: 414-420.

17. Gonzalez-Perez J, Villa-Collar C, Sobrino Moreiras T, et al. Tear film inflammatory mediators during continuous wear of contact lenses and corneal refractive therapy. Br J Ophthalmol 2012;96:1092-1098.

18. Matsubara M, Kamei Y, Takeda S, et al. Histologic and histochemical changes in rabbit cornea produced by an orthokeratology lens. Eye Contact Lens 2004;30:198-204. Discussion 205-196.

19. Cheah P-S, Norhani M, Bariah M-A, et al. Histomorphometric profile of the corneal response to short-term reverse-geometry orthokeratology lens wear in primate corneas - a pilot study. Cornea 2008;27:461-470.

20. Choo JD, Caroline PJ, Harlin DD, et al. Morphologic changes in cat epithelium following overnight lens wear with the paragon CRT lens for corneal reshaping. Invest Ophthalmol Vis Sci 2004;45:U578.

21. Carracedo G, Gonzalez-Meijome JM, Pintor J. Changes in diadenosine polyphosphates during alignment-fit and orthokeratology rigid gas permeable lens wear. Invest Ophthalmol Vis Sci 2012;53:4426-4432.

22. Gilbard JP, Gray KL, Rossi SR. A proposed mechanism for increased tearfilm osmolarity in contact lens wearers. Am $J$ Ophthalmol 1986;102: 505-507.

23. Nichols JJ, Sinnott LT. Tear film, contact lens, and patient-related factors associated with contact lens-related dry eye. Invest Ophthalmol Vis Sci 2006;47:1319-1328

24. Efron N, Jones L, Bron AJ, et al. The TFOS international Workshop on contact lens discomfort: report of the contact lens interactions with the ocular surface and adnexa subcommittee. Invest Ophthalmol Vis Sci 2013; $54: 98-122$

25. Lemp MA, Baudouin C, Baum J, et al. The definition and classification of dry eye disease: report of the definition and classification subcommittee of the international dry eye WorkShop (2007). Ocul Surf 2007;5:75-92.

26. Johnson ME, Murphy PJ. The effect of instilled fluorescein solution volume on the values and repeatability of TBUT measurements. Cornea 2005;24: 811-817.

27. Patel S, Farrell J, Blades KJ, et al. The value of a phenol red impregnated thread for differentiating between the aqueous and non-aqueous deficient dry eye. Ophthalmic Physiol Opt 1998;18:471-476.

28. Nichols KK, Nichols JJ, Mitchell GL. The reliability and validity of McMonnies dry eye index. Cornea 2004;23:365-371.

29. Gonzalez-Mesa A, Villa-Collar C, Lorente-Velazquez A, et al. Anterior segment changes produced in response to long-term overnight orthokeratology. Curr Eye Res 2013;38:862-870.

30. Tomlinson A, McCann LC, Pearce EI. Comparison of human tear film osmolarity measured by electrical impedance and freezing point depression techniques. Cornea 2010;29:1036-1041.

31. Benelli U, Nardi M, Posarelli C, et al. Tear osmolarity measurement using the TearLab (TM) Osmolarity System in the assessment of dry eye treatment effectiveness. Contact Lens Anterior Eye 2010;33:61-67.

32. Khanal S, Millar TJ. Barriers to clinical uptake of tear osmolarity measurements. Br J Ophthalmol 2012;96:341-344.

33. Tomlinson A, Khanal S, Ramaesh K, et al. Tear film osmolarity: determination of a referent for dry eye diagnosis. Invest Ophthalmol Vis Sci 2006; 47:4309-4315.

34. Sarac O, Gurdal C, Bostanci-Ceran B, et al. Comparison of tear osmolarity and ocular comfort between daily disposable contact lenses: hilafilcon B hydrogel versus narafilcon A silicone hydrogel. Int Ophthalmol 2012;32:229-233.

35. Alghamdi WM, Markoulli M, Holden BA, et al. Impact of duration of contact lens wear on the structure and function of the meibomian glands. Ophthalmic Physiol Opt 2016;36:120-131.

36. Liu H, Begley C, Chen M, et al. A link between tear instability and hyperosmolarity in dry eye. Invest Ophthalmol Vis Sci 2009;50:3671-3679.

37. Gipson IK, Argueso P, Beuerman R, et al. Research in dry eye: report of the Research Subcommittee of the international dry eye WorkShop (2007). Ocul Surf 2007;5:179-193.

38. Linna TU, Vesaluoma MH, Pérez-Santonja JJ, et al. Effect of myopic LASIK on corneal sensitivity and morphology of subbasal nerves. Invest Ophthalmol Vis Sci 2000;41:393-397.

39. Eperjesi F, Aujla M, Bartlett H. Reproducibility and repeatability of the OcuSense TearLab osmometer. Graefes archive Clin Exp Ophthalmol 2012;250:1201-1205. 